\title{
Electrochemical oxidation of substituted benzylamines in aquo-acetic acid medium: substituent and solvent effects
}

\author{
A THIRUMOORTHI ${ }^{1}$ and $\mathrm{K}$ P ELANGO ${ }^{2, *}$ \\ ${ }^{1}$ Department of Chemistry, Karpagam College of Engineering, Coimbatore 641032 \\ ${ }^{2}$ Department of Chemistry, Gandhigram Rural University, Gandhigram 624302 \\ e-mail: drkpelango@rediffmail.com
}

MS received 9 June 2006; revised 14 May 2007

\begin{abstract}
Electrochemical oxidation of nine para- and meta-substituted benzylamines in varying mole fractions of acetic acid in water has been investigated in the presence of $0 \cdot 1 \mathrm{M}$ sulphuric acid as supporting electrolyte. The oxidation potentials correlate well with Hammett's substituent constants affording negative reaction constants. The correlation of potential values with macroscopic solvent parameters is non-linear suggesting that the operation of both specific and non-specific solvent-solvent-solute interaction mechanisms. Multiple correlation analysis of the experimental data with Kamlet-Taft solvatochromic parameters is employed.
\end{abstract}

Keywords. Solvent effect; benzylamine; electrochemical oxidation; cyclic voltammetry.

\section{Introduction}

Aromatic amines and its derivatives are used as intermediates for the manufacturing of various organic compounds such as colorants, agrochemicals, pharmaceutical agents, etc. ${ }^{1}$ The compounds have been found to be widely distributed in aqueous environments and cause teratosis in aquatic species. ${ }^{2}$ Aromatic amine is carcinogenic ${ }^{3}$ and also reacts easily in the blood to convert hemoglobin into methemoglobin, preventing oxygen uptake. ${ }^{4}$ Therefore, a serious effect on human health over a long period of time is possible, even if aromatic amine is in low concentrations.

Currently several treatment methods for wastewater containing aromatic amines have been developed including biodegradation, ${ }^{5}$ catalytic oxidation, ${ }^{6}$ separation using membranes, ${ }^{7,8}$ emulsion liquid membranes, ${ }^{9}$ ultrasonic degradation, ${ }^{10}$ decomposition in supercritical water, ${ }^{11}$ adsorption using activated charcoal, ${ }^{12}$ as well as an electrochemical (EC) method $^{13}$. The EC method has some advantages over chemical or biological methods. Chemical methods require a large amount of reactive chemical reagents which can often cause secondary pollution. On the other hand, biological methods are temperature-

\footnotetext{
*For correspondence
}

dependent and are inhibited by the toxicity of pollutants. ${ }^{14}$ EC methods have little or no harmful effects on the environment as they do not involve the use of harmful reagents. EC methods are clean and easy to handle and therefore, there is an increasing interest in the development of practical EC methods for treatment of high toxicity and biorefractory organic pollutants.

Furthermore, the study of solute-solvent interactions in binary mixtures is more complex than in pure solvents. In a pure solvent the composition of the microsphere of solvation of a solute, the so called cybotatic region, is the same as in the bulk solvent but in binary mixtures the composition of this microsphere can be different. The solute can interact to different degrees with the components of the mixture, and this difference is reflected in the composition of the microsphere of solvation. The effect of varying the composition of the mixture from the bulk solvent to the solvation sphere is called preferential solvation. ${ }^{15}$

The objective of the present work includes a systematic study of the substituent and solvent effects on electrochemical oxidation of a series of para- and meta-substituted benzylamines, in varying mole fractions of co-solvent acetic acid in water, and the analysis of the data using linear free energy relationships with an aim to get better insight into the mechanism of benzylamine oxidation. 


\section{Experimental}

\subsection{Materials and methods}

All chemicals and solvents used were of analytical grade (Aldrich, Merck or SRL-India). The solid benzylamines were used as such and the liquid benzylamines were used after vacuum distillation. Acetic acid was purified by reported method. ${ }^{16}$ Doublydistilled water was used throughout the work.

Solutions of electrolyte were prepared with doubly distilled water. All electrochemical experiments were performed at room temperature using a standard three-electrode, two compartment configuration with a Glassy Carbon (GC) working electrode, a spiral platinum counter electrode and a $\mathrm{Ag} \mid \mathrm{AgCl}(\mathrm{KCl}$ sat.) reference electrode. The carbon electrodes were polished between experiments with aluminium oxide (alumina) paste. The cyclic voltammetric experiments were carried out with a computer-controlled electrochemical system (CHI643B Electrochemical Analyzer) at $50 \mathrm{mV} \mathrm{s}^{-1}$. All solutions were de-oxygenated thoroughly by purging with nitrogen gas.

Correlation analyses were carried out using Microcal Origin (version 6) computer software. The goodness of fit was discussed using correlation coefficient ( $r$ in the case of simple linear regression and $R$ in the case of multiple linear regression), standard deviation, sd and Exner's statistical parameters, $\psi{ }^{17}$ The percentage contribution $\left(P_{x}\right)$ of a parameter to the total effect on reactivity was computed using the regression co-efficient of each parameter as reported earlier. ${ }^{18}$

\section{Results and discussion}

A cyclic voltammetric study of $1 \mathrm{mM}$ solution of benzylamine in varying mole fractions of co-solvent acetic acid in water with $0.1 \mathrm{M}$ sulphuric acid was carried out at the GC electrode. A typical voltammogram obtained is shown in figure 1. During the forward scan, a single peak appears at $0.897 \mathrm{~V}$ versus standard $\mathrm{Ag} \mid \mathrm{AgCl}$ ( $\mathrm{KCl}$ sat.) as the reference electrode. On the reverse scan, one reduction peak is observed. The formation of a single peak during the first forward scan (oxidation) can be easily explained on the basis of the mechanism proposed by Adams. ${ }^{19}$ According to this mechanism, benzylamine during oxidation loses one electron to produce a cation radical (scheme 1) and undergoes further oxidation to give the product, at the same potential. Thus the overall process consists of a single step two-electron oxidation of benzylamine. This accounts for the observation of a single oxidation peak during the first forward scan. Parallel observations have been reported in literature for electrochemical oxidation of benzylamine at a polycrystalline gold electrode in solution of $0.04 \mathrm{M} \mathrm{KClO}_{4}$ or $0.04 \mathrm{M} \mathrm{HClO}_{4}{ }^{20}$

The results in table 1 indicate that on increasing the mole fraction of acetic acid in the mixture, there is a decrease in polarity and the oxidation becomes more difficult. This suggests that the transition state is more polar than the reactant (scheme 1). Hence, a decrease in polarity of the medium destabilizes such a transition state and consequently makes oxidation difficult, which requires more potential.

\subsection{Structure-reactivity correlation}

The effect of substituents on oxidation potential was studied with nine para- and meta-substituted ben-

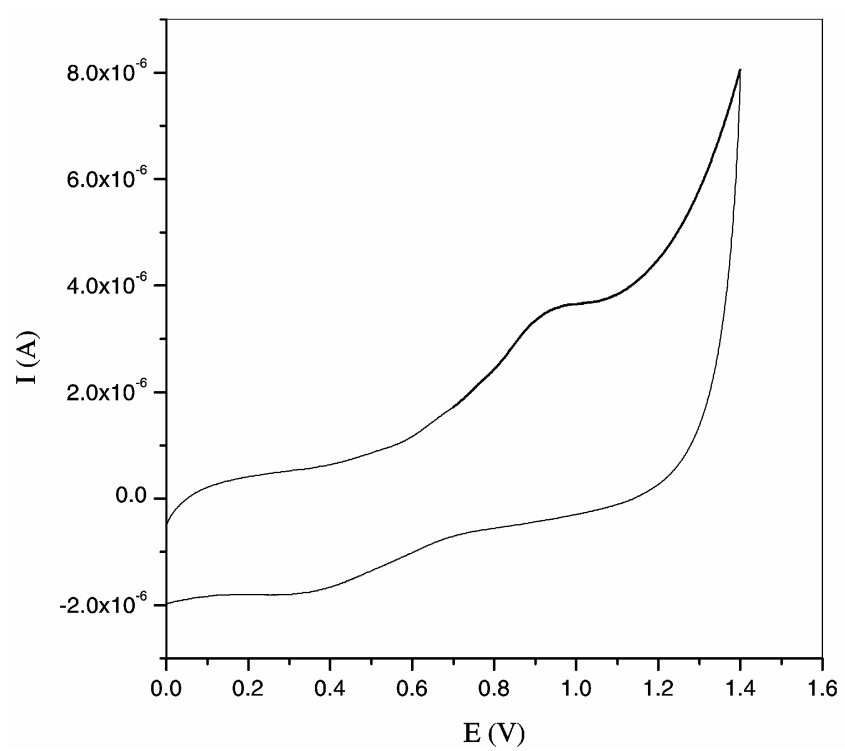

Figure 1. Cyclic voltammogram obtained for $1 \mathrm{mM}$ benzylamine in 0.1 mole fraction of acetic acid in water containing $0 \cdot 1 \mathrm{M}$ sulphuric acid at a scan rate of $0.05 \mathrm{~V} \mathrm{~s}^{-1}$.

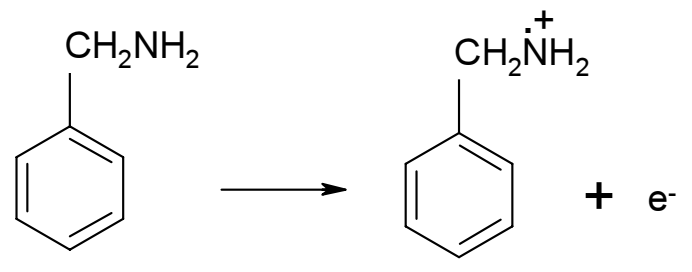

Scheme 1. 
Table 1. Electrochemical data $\left(E_{\mathrm{p}}[\mathrm{V}]\right)$ from $\mathrm{CV}$ for oxidation of benzylamines in varying mole fractions of acetic acid in water.

\begin{tabular}{|c|c|c|c|c|c|c|c|c|c|}
\hline \multirow{2}{*}{$\begin{array}{l}\text { Substituents } \\
\text { in benzylamine moiety }\end{array}$} & \multicolumn{9}{|c|}{ Mole fraction of acetic acid } \\
\hline & $0 \cdot 1$ & $0 \cdot 2$ & $0 \cdot 3$ & $0 \cdot 4$ & 0.5 & $0 \cdot 6$ & 0.7 & 0.8 & $0 \cdot 9$ \\
\hline $\mathrm{H}$ & 0.897 & 0.898 & 0.900 & 0.903 & 0.909 & 0.936 & 0.959 & 0.982 & 1.009 \\
\hline$m$-OMe & 0.991 & 1.006 & 1.015 & 1.047 & 1.050 & 1.059 & 1.076 & 1.088 & 1.097 \\
\hline$m-\mathrm{Me}$ & 0.921 & 0.941 & 0.956 & 0.962 & 0.982 & 0.994 & 1.003 & 1.015 & 1.027 \\
\hline$m-\mathrm{Cl}$ & 0.980 & 1.018 & 1.024 & 1.044 & 1.085 & 1.094 & $1 \cdot 103$ & $1 \cdot 109$ & $1 \cdot 123$ \\
\hline$p$-OMe & 0.912 & 0.918 & 0.924 & 0.936 & 0.947 & 0.956 & 0.971 & 0.985 & 1.000 \\
\hline$p$-Me & 0.947 & 0.956 & 0.962 & 0.974 & 0.980 & 0.985 & 0.991 & 1.006 & 1.021 \\
\hline$p-\mathrm{F}$ & 0.959 & 0.971 & 0.991 & 1.006 & 1.021 & 1.035 & 1.041 & 1.065 & 1.083 \\
\hline$p-\mathrm{Cl}$ & 0.988 & 0.997 & 1.015 & 1.030 & 1.050 & 1.074 & 1.094 & $1 \cdot 100$ & $1 \cdot 103$ \\
\hline$p-\mathrm{COOH}$ & 1.030 & 1.041 & 1.053 & 1.059 & 1.076 & 1.082 & 1.096 & $1 \cdot 106$ & $1 \cdot 112$ \\
\hline
\end{tabular}

Table 2. The oxidation potential data $\left(\Delta \log E_{\mathrm{X}}[\mathrm{V}]\right)$ for the substituents.

\begin{tabular}{|c|c|c|c|c|c|c|c|c|c|}
\hline \multirow{2}{*}{$\begin{array}{l}\text { Substituents } \\
\text { in benzylamine moiety }\end{array}$} & \multicolumn{9}{|c|}{ Mole fraction of acetic acid } \\
\hline & $0 \cdot 1$ & $0 \cdot 2$ & $0 \cdot 3$ & $0 \cdot 4$ & $0 \cdot 5$ & $0 \cdot 6$ & 0.7 & $0 \cdot 8$ & $0 \cdot 9$ \\
\hline$m$-OMe & -1.551 & $-1 \cdot 821$ & $-2 \cdot 546$ & $-2 \cdot 428$ & $-2 \cdot 378$ & $-2 \cdot 074$ & -1.973 & -1.787 & -1.484 \\
\hline$m$-Me & -0.405 & -0.725 & -0.944 & -0.995 & -1.231 & -0.978 & -0.742 & -0.556 & $-0 \cdot 303$ \\
\hline$m-\mathrm{Cl}$ & $-1 \cdot 366$ & $-2 \cdot 024$ & $-2 \cdot 091$ & $-2 \cdot 378$ & $-2 \cdot 968$ & $-2 \cdot 664$ & $-2 \cdot 428$ & $-2 \cdot 142$ & -1.922 \\
\hline$p$-OMe & $-0 \cdot 253$ & $-0 \cdot 337$ & $-0 \cdot 405$ & -0.556 & $-0 \cdot 641$ & $-0 \cdot 337$ & $-0 \cdot 202$ & $-0 \cdot 051$ & $-0 \cdot 152$ \\
\hline$p$-Me & -0.843 & -0.978 & -1.045 & $-1 \cdot 197$ & $-1 \cdot 197$ & -0.826 & -0.539 & -0.405 & $-0 \cdot 202$ \\
\hline$p-\mathrm{F}$ & -1.045 & $-1 \cdot 231$ & -1.531 & -1.731 & -1.889 & -1.669 & $-1 \cdot 383$ & $-1 \cdot 399$ & -1.248 \\
\hline$p-\mathrm{Cl}$ & -1.534 & -1.669 & -1.939 & $-2 \cdot 142$ & $-2 \cdot 378$ & $-2 \cdot 327$ & $-2 \cdot 276$ & -1.990 & -1.585 \\
\hline$p-\mathrm{COOH}$ & $-2 \cdot 243$ & $-2 \cdot 411$ & $-2 \cdot 580$ & $-2 \cdot 630$ & $-2 \cdot 816$ & $-2 \cdot 462$ & $-2 \cdot 310$ & $-2 \cdot 091$ & -1.737 \\
\hline
\end{tabular}

zylamines in varying mole fraction of acetic acid in water. The oxidation potential was measured for each substituent by cyclic voltammetry using the same scan rate, working electrode, electrolyte concentration, and compound concentration. The difference in oxidation potentials can be calculated using the following (1), as described earlier. ${ }^{21}$

$$
\Delta E_{\mathrm{X}}=E_{\mathrm{paH}}-E_{\mathrm{paX}}
$$

where $E_{\mathrm{paH}}$ is the oxidation potential for unsubstituted benzylamine and $E_{\mathrm{paX}}$ is that of $\mathrm{X}$-substituted benzylamine. The reaction constant $\rho$ for the electrode process can then be determined by plotting $\Delta \log k_{\mathrm{X}}$ ( $\Delta \log E_{\mathrm{X}}$ in the present case) against substituent constants $\sigma(2){ }^{21}$

$$
\Delta \log E_{\mathrm{X}}=\rho \sigma
$$

The values of $\Delta \log E_{\mathrm{X}}$ for the first oxidation potentials of all the substituted benzylamines (table 2), in all mole fractions of the solvent mixture, showed a linear relationship with respect to Hammett's substituent constants ${ }^{17}$ and a typical plot is shown in figure 2. The statistical results for these plots are given in table 3 . The values of the reaction constant, $\rho$, were found to be negative, which confirms the formation of a positively charged transition state. The magnitude of reaction constants obtained in the present study is lesser when compared to that for the electrochemical oxidation of anilines in acetic acidwater media $(\rho>-5 \cdot 26){ }^{22}$ This may be due to the fact that structural changes in the side chain may be expected to affect the reaction constants. ${ }^{23}$ Introduction of methylene group between the reaction site and the benzene ring might have reduced the transmission of the electrical effect of substituents through the side chain and hence the observed low reaction constants.

\subsection{Solvent-reactivity correlation}

The electro-oxidation of benzylamine has been studied in nine mole fractions of acetic acid in water ranging 
Table 3. Results of simple linear correlation of the oxidation potential data, $\Delta \log E_{\mathrm{X}}$, with substituent constants as a function of relative permittivity of the medium.

\begin{tabular}{|c|c|c|c|c|c|}
\hline Mole fraction of acetic acid in water & $\varepsilon_{r}$ & $100 r^{2}$ & $\mathrm{~s} d$ & $\psi$ & $\rho$ \\
\hline $0 \cdot 1$ & $71 \cdot 17$ & 89 & $0 \cdot 316$ & $0 \cdot 51$ & $-2 \cdot 31 \pm 0 \cdot 47$ \\
\hline $0 \cdot 2$ & 63.94 & 96 & $0 \cdot 223$ & $0 \cdot 34$ & $-2 \cdot 64 \pm 0 \cdot 33$ \\
\hline $0 \cdot 3$ & $56 \cdot 72$ & 89 & $0 \cdot 394$ & 0.53 & $-2 \cdot 76 \pm 0.58$ \\
\hline 0.4 & $49 \cdot 49$ & 93 & $0 \cdot 305$ & 0.43 & $-2 \cdot 79 \pm 0 \cdot 45$ \\
\hline 0.5 & $42 \cdot 27$ & 97 & $0 \cdot 216$ & $0 \cdot 27$ & $-3 \cdot 21 \pm 0 \cdot 32$ \\
\hline $0 \cdot 6$ & $35 \cdot 05$ & 97 & $0 \cdot 241$ & $0 \cdot 30$ & $-3 \cdot 63 \pm 0.36$ \\
\hline 0.7 & $27 \cdot 82$ & 96 & $0 \cdot 275$ & $0 \cdot 33$ & $-3 \cdot 35 \pm 0.41$ \\
\hline $0 \cdot 8$ & $20 \cdot 60$ & 95 & $0 \cdot 274$ & $0 \cdot 35$ & $-3 \cdot 16 \pm 0 \cdot 41$ \\
\hline 0.9 & $13 \cdot 37$ & 94 & $0 \cdot 271$ & $0 \cdot 39$ & $-2.74 \pm 0.40$ \\
\hline
\end{tabular}

$\varepsilon_{r}$, Relative permittivity of the medium; $100 r^{2}$, Explained variance; sd, Standard deviation; $\psi$, Exner's statistical parameter; $\rho$, Reaction constant

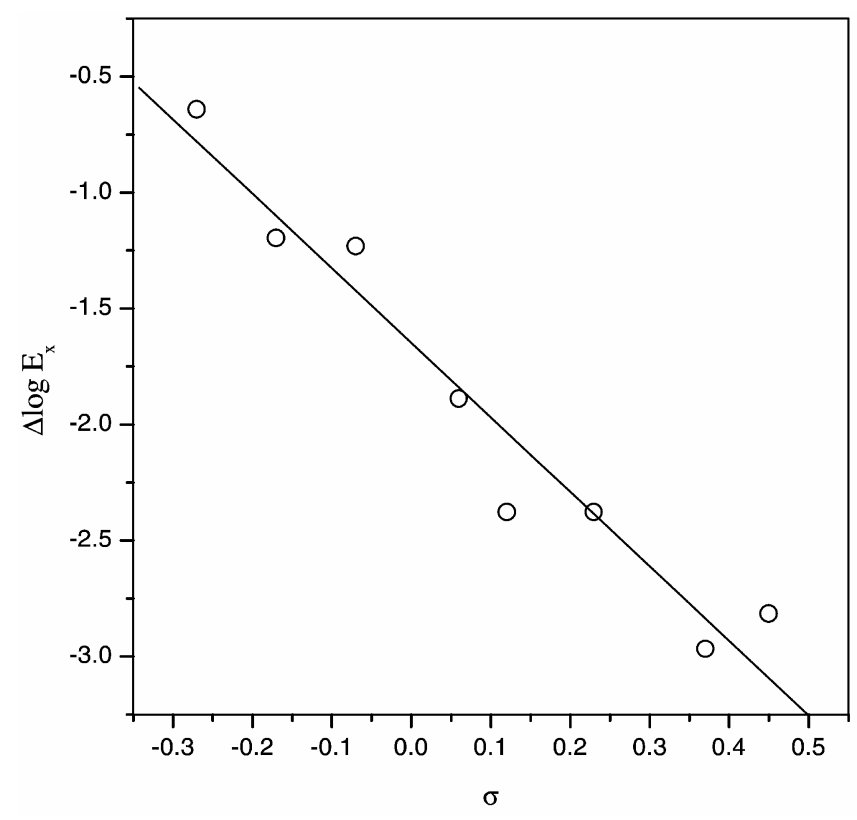

Figure 2. Correlation of $\Delta \log k_{x}$ with $\sigma$ (Hammett) for various substituents solvent: 0.5 mole fraction of acetic acid in water.

from $0 \cdot 1$ to 0.9 (table 1 ). A typical cyclic voltammogram for the oxidation of the benzylamine in varying mole fractions of acetic acid in water is shown in figure 3. Curvilinear plots are obtained for the correlation of oxidation potential data with the inverse of relative permittivity of the medium through the Laidler-Erying ${ }^{24}$ equation. A representative plot is shown in figure 4. Likewise, the correlation of oxidation potential data with the GrunwaldWinstein $^{25}$ solvent ionizing power, $Y$, is also nonlinear. The solvent parameter values used in the present study viz. relative permittivity ${ }^{26}$ and ionising power $^{25}$ of the mixtures employed was calculated as

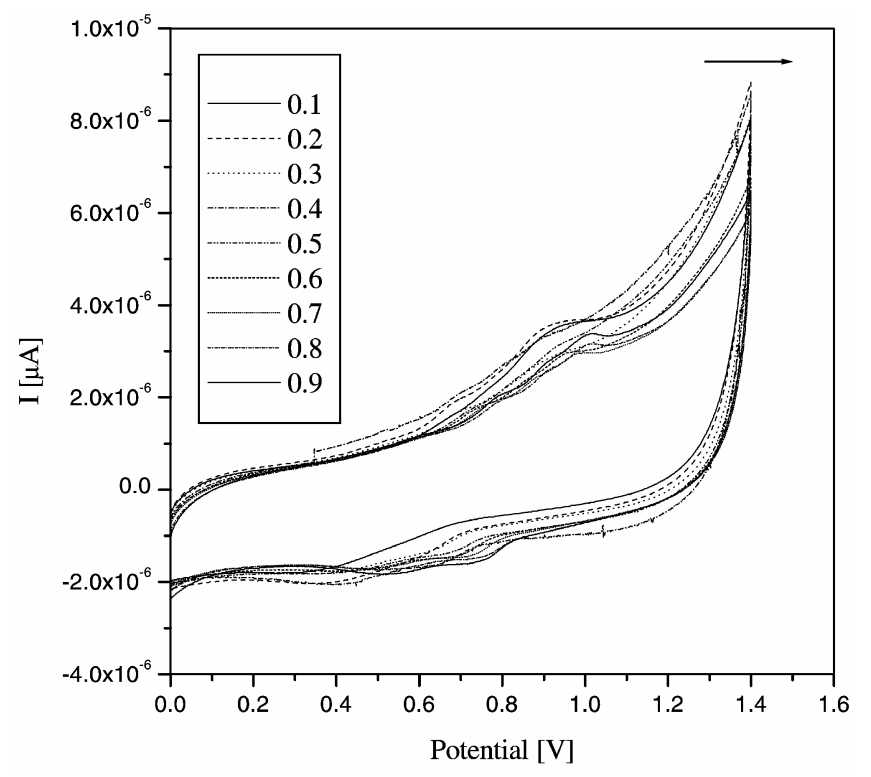

Figure 3. Cyclic voltammograms obtained for benzylamine in varying mole fractions of acetic acid in water.

described in literature. A representative plot is shown in figure 5 .

Such poor correlations observed in the present study indicate that no single macroscopic solvent parameter such as relative permittivity or ionizing power can possibly account for the multitude of solutesolvent interactions on the molecular microscopic level. Thus these bulk properties will poorly describe the micro-environment around the reacting species, which governs the stability of the transition state. Hence, the operation of selective or preferential solvation, which includes both non-specific solutesolvent association caused by dielectric enrichment 
Table 4. Statistical results and weighted percentage contributions for the correlation of log Ep for the electrochemical oxidation of substituted benzylamines with Kamlet-Taft's solvatochromic parameters $\alpha, \beta$ and $\pi^{*}$.

\begin{tabular}{|c|c|c|c|c|c|c|c|c|c|}
\hline Substituent & $100 R^{2}$ & $\mathrm{~s} d$ & $\psi$ & $a$ & $b$ & $s$ & $P_{\alpha}$ & $P_{\beta}$ & $P_{\pi^{*}}$ \\
\hline $\mathrm{H}$ & 89 & 0.008 & $0 \cdot 39$ & $-0.38 \pm 0.37$ & $-0.96 \pm 0.82$ & $-0.05 \pm 0.06$ & 27 & 70 & 03 \\
\hline$m$-OMe & 98 & 0.003 & $0 \cdot 16$ & $-0.11 \pm 0.12$ & $-0.33 \pm 0.26$ & $-0.06 \pm 0.02$ & 22 & 65 & 13 \\
\hline$m$-Me & 99 & 0.001 & $0 \cdot 13$ & $-0.11 \pm 0.07$ & $-0 \cdot 38 \pm 0 \cdot 16$ & $-0 \cdot 14 \pm 0 \cdot 01$ & 17 & 61 & 22 \\
\hline$m-\mathrm{Cl}$ & 97 & 0.004 & 0.09 & $0.42 \pm 0.20$ & $1.06 \pm 0.46$ & $-0.20 \pm 0.03$ & 25 & 63 & 12 \\
\hline$p$-OMe & 99 & 0.002 & $0 \cdot 11$ & $-0.14 \pm 0.09$ & $-0.32 \pm 0.21$ & $-0.07 \pm 0.01$ & 26 & 61 & 13 \\
\hline$p$-Me & 99 & 0.001 & 0.05 & $-0.20 \pm 0.02$ & $-0.38 \pm 0.05$ & $-0.05 \pm 0.01$ & 32 & 60 & 08 \\
\hline$p-\mathrm{F}$ & 99 & 0.001 & $0 \cdot 19$ & $-0.10 \pm 0.05$ & $-0 \cdot 13 \pm 0.11$ & $-0.12 \pm 0.01$ & 30 & 37 & 33 \\
\hline$p-\mathrm{Cl}$ & 99 & $0 \cdot 002$ & $0 \cdot 13$ & $0 \cdot 28 \pm 0 \cdot 12$ & $0.56 \pm 0.27$ & $-0.15 \pm 0.02$ & 28 & 57 & 15 \\
\hline$p$ - $\mathrm{COOH}$ & 99 & 0.002 & 0.13 & $0.02 \pm 0.08$ & $0.07 \pm 0.18$ & $-0.08 \pm 0.01$ & 11 & 40 & 49 \\
\hline
\end{tabular}

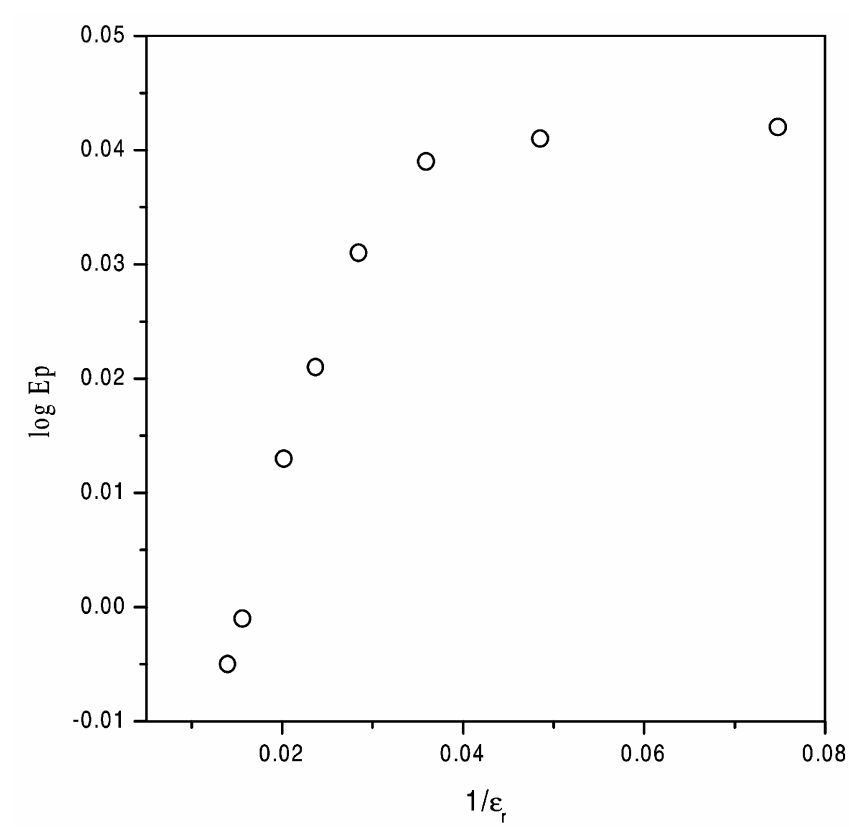

Figure 4. Laidler-Eyrng plot for the electrochemical oxidation of $p$-chlorobenzylamine in acetic acid-water media.

in the solvation shell of solute ions or dipolar solute molecules and specific solute-solvent association such as hydrogen bonding or electron pair donor/ electron pair acceptor interactions, is likely in the present case. ${ }^{16}$

The influence of specific and non-specific solutesolvent-solvent interactions on the reactivity was studied by employing the Kamlet-Taft solvatochromic method. ${ }^{27}$ The correlation of oxidation potential data with solvatochromic parameters $\alpha, \beta$ and $\pi^{*}$ is excellent. The correlation suggests the operation of both specific and non-specific solute-solvent interactions which govern the reactivity. Further, results of the multiple correlation and the weighted percentage contributions of the solvatochromic parame-

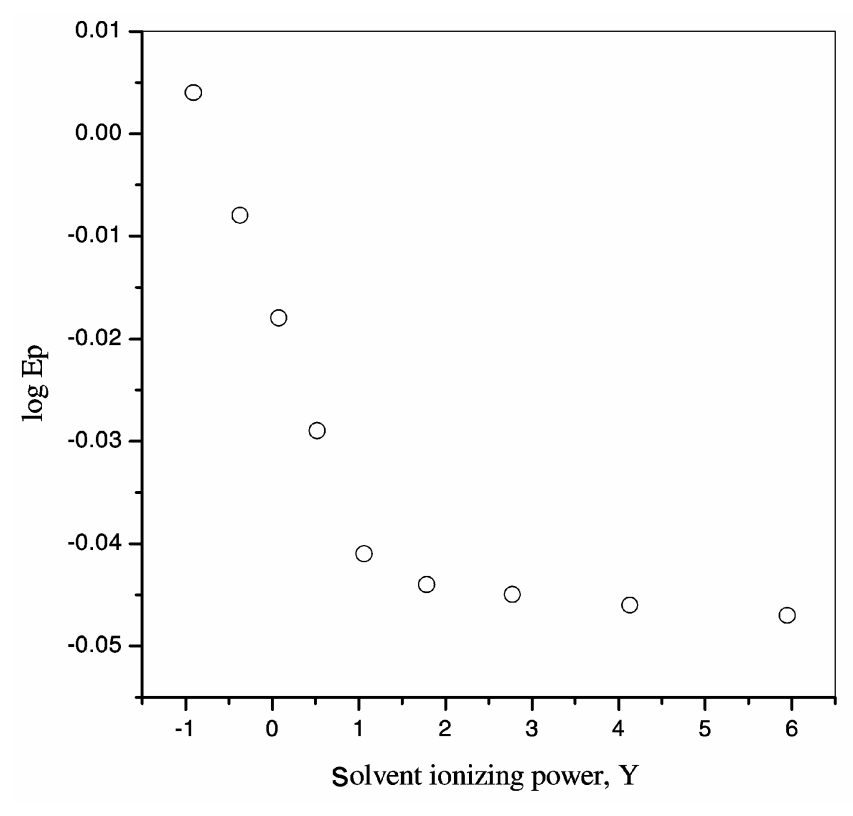

Figure 5. Grunwald-Winstein plot for the electrochemical oxidation of benzylamine in acetic acid-water media.

ters (table 4) indicate that the specific solventsolvent-solute interactions, as indicated by $P_{\alpha}$ and $P_{\beta}$, play a major role in governing the oxidation process. These two parameters alone accounts for about $80 \%$ of the observed solvent effect. Further, the contribution of hydrogen bond acceptor basicity (represented by $\beta$ term) to the total solvent effect was found to be predominant. The signs of the coefficients of these two terms are randomly observed and this may be due to complexing interactions between solute and the solvent.

However, the sign of the coefficients of the polarity/polarizability term, $\pi^{*}$, is negative. The negative sign of the coefficients of this term also suggests that with decrease in polarizability/dipolarity of the 
medium makes the oxidation process difficult. Increase in mole fraction of acetic acid in the mixture decreases the polarity/polarizability of the medium and consequently increases the oxidation potential and hence the oxidation becomes more difficult. This suggests that the transition state is more polar than the reactant. Hence an increase in polarity of the medium stabilizes such a transition state and consequently makes the oxidation easier, which requires less potential.

It may be concluded that since amines are very harmful to human health and the environment, the removal of amines from the environment is the ultimate goal of research today. For this a deep understanding of the mechanism of the oxidation process of amines are needed. In this work we have studied the detailed mechanism of electrochemical oxidation of some para- and meta-substituted benzylamines. The results of solvent effects indicate that an increase in polarity of the medium makes the oxidation process easier. Further, the reactivity is influenced both by non-specific and specific solute-solvent interactions. The correlation of oxidation potential data with substituent constants reveals that a positively charged transition state is formed during the oxidation process.

\section{Acknowledgement}

We thank Dr S Abraham John, Lecturer in Chemistry, Gandhigram Rural University, Gandhigram for providing Cyclic Voltammetry instrument and also for scientific discussions during the preparation of the manuscript.

\section{References}

1. Zok S, Gorege G, Kalsch W and Nagel R 1991 Sci. Total Environ. 109/110 411

2. Abram F S H and Sims I R 1982 Water Res. 161309
3. Walpole A L and Williams M H C 1958 Brit. Med. Bull. 14141

4. Beck W S 1998 Hematology (Cambridge: The MIT Press) p. 184

5. O’Neill F J, Bromley Challenor K C A, Greenwood R J and Knapp J S 2000 Water Res. 344397

6. Barbier J, Oliverio L, Renard B and Duprez D 2002 Catal. Today. 7529

7. Pithan F, Bickel C S and Lichtenthaler R N 2002 Desalination 1481

8. Ferreira F C, Han S, Boam A, Zhang S and Livingston A G 2002 Desalination 148267

9. Devulapalli R and Jones F 1999 J. Hazard. Mater. B70 157

10. Jiang Y, Petrier C and Waite T D 2002 Ultrasonics Sonochem. 9163

11. Qi X H, Zhuang Y Y, Yuan Y C and Gu W X $2002 \mathrm{~J}$. Hazard. Mater. 9051

12. Zhou M L, Martin G, Taha S and Sant'Anna F 1998 Water Res. 321109

13. Brillas E and Casado J 2002 Chemosphere 47241

14. Matsushita M, Kuramitz H and Tanaka S 2005 Environ. Sci. Technol. 393805

15. Bosch E, Rived F and Roses M 1996 J. Chem. Soc. Perkin Trans. 22177

16. Reichardt C 1988 Solvents and solvent effects in organic chemistry (Weinheim: $\mathrm{VCH}$ )

17. Shorter J 1982 Correlation analysis of organic reactivity (Letchworth: Research Studies Press)

18. Reichardt C 1979 Angew. Chem. Int. Ed. Engl. 1898

19. Adams R N 1969 Electrochemistry at solid electrodes (New York: Marcel Dekker)

20. Luczak T, Brzezinska M B, Bron M and Holze R 1997 Vibra. Spect. 1517

21. Glatzhofer D T and Morvant M C 1998 J. Phys. Org. Chem. 11731

22. Bhuvaneshwari D S and Elango K P 2006 Z. Naturforsch. B61 1254

23. Jaffe H H 1953 Chem. Rev. 191

24. Amis E S and Hinton J I 1973 Solvent effect of chemical phenomena (New York: Academic Press)

25. Fainberg A H and Winstein S $1956 \mathrm{~J}$. Am. Chem. Soc. 782770

26. Marcus Y 1985 Ion solvation (New York: WileyInterscience)

27. Kamlet M J, Abboud J M, Abraham M H and Taft R W 1983 J. Org. Chem. 482877 